\title{
De l'importance des observations pour la prévision numérique du temps
}

\author{
The importance of observations for numerical weather prediction
}

\author{
par Philippe Courtier, \\ Météo-France
}

In meteorology, and more generally in physics, the observations are used in order to understand, in order to predict, in order to validate modelling. But the computational weather forecast has some specificities.

En météorologie, comme plus généralement en physique, les observations servent à comprendre, servent à prévoir, servent à valider les modèles développés. Nous allons voir ce qui est spécifique de la prévision numérique du temps.

\section{I $\square$ DES MODÈLES COMPLEXES}

La prévision numérique du temps repose sur l'intégration de modèles numériques qui sont fondés sur la discrétisation des équations de Navier-Stokes. Les principales approximations mises en cuvre sont les suivantes :

- l'atmosphère est supposée être le mélange de deux gaz parfaits, l'un diatomique, l'air sec, et le second triatomique, la vapeur d'eau. Par ailleurs elle comprend des composés minoritaires, négligeables sur le plan de la dynamique des fluides, mais fondamentaux pour leur propriétés radiatives, les principaux étant le gaz carbonique et l'ozone.

- L'approximation de couche mince est faite pour une planète terre supposée sphérique. Cela signifie que l'écoulement, bien que tridimensionnel, se situe à une même distance du centre de la terre. Ou encore que les équipotentielles du champ de gravité sont des sphères infiniment proches les unes des autres comparativement au rayon de la terre.

- L'hypothèse hydrostatique est faite sur la verticale, ce qui revient à négliger les accélérations verticales, mais non les mouvements verticaux. Cette approximation est excellente pour les mouvements dont l'échelle horizontale est supérieure à l'échelle verticale caractéristique de l'atmosphère qui est typiquement de $10 \mathrm{~km}$.

La partie dynamique décrite ci-dessus est complétée d'une partie dite physique qui, pour l'essentiel, paramétrise les phénomènes de sous-maille :

- Le rayonnement dans les courtes longueurs d'onde, principalement le visible, constitue le moteur de l'atmosphère au travers du chauffage solaire.

- L'atmosphère rayonne comme un corps gris dans les longueurs d'onde infrarouge où les gaz à effet de serre jouent tout leur rôle. Tant dans les courtes que longues longueurs d'onde, l'interaction entre les nuages et le rayonnement est particulièrement délicate à modéliser.

- La couche limite planétaire, qui est une couche limite au sens classique du terme, doit représenter les interactions turbulentes entre l'atmosphère et la surface dans les premières centaines de mètres.

- La formation des nuages dans les deux types observés, à savoir stratiformes (étalés sur l'horizontale) ou cumuliformes (à fort développement vertical), et donc la condensation et la pluie sont d'une importance énergétique majeure.

- La description des cycles énergétiques et de l'eau des sols. - La représentation de l'orographie et ses interactions avec l'écoulement, en particulier la traînée des ondes de gravité qui est un phénomène de dissipation rapide avec des ondes formées au voisinage de reliefs qui se propagent verticalement et déferlent à un niveau critique vers une dizaine de $\mathrm{km}$; ou encore le piégeage de masse d'air froide dans les vallées l'hiver.

Le développement de ces modèles est une science jeune ayant suivi le développement des calculateurs, mais aussi le développement de la météorologie qui n'est réellement passé du stade de science descriptive à celui de science prédictive et 
quantitative que vers la moitié du vingtième siècle. Par exemple les papiers fondateurs de l'instabilité barocline (troisième instabilité hydrodynamique des écoulements subsoniques avec l'instabilité barotrope et l'instabilité convective) date du milieu du siècle. Or elle est à la base de la formation des perturbations et tempêtes des latitudes tempérées. Les modèles sont ainsi validés au travers de campagnes de mesures géophysiques organisées le plus souvent à l'échelle internationale pour comprendre tel ou tel phénomène encore mal cerné.

La dimension de la variable d'état des modèles actuellement opérationnels dans les grands centres de prévision numérique est comprise entre 107 et 108 . Cela implique l'utilisation des plus puissants calculateurs disponibles mêlant parallélisme vectoriel et scalaire optimisé en fonction de l'architecture des calculateurs.

En résumé, nous sommes face à un problème de grande taille, fortement non-linéaire et avec un spectre très raide. Cependant, tout n'est pas perdu car nous en savons un peu plus.

\section{II — CHAOS DÉTERMINISTE}

Les solutions météorologiquement admissibles des équations ci-dessus ne sont pas toutes les solutions. En effet l'atmosphère est un système dynamique à la fois forcé et dissipatif et qui présente un attracteur. A grande échelle, on peut donner quelques propriétés de cet attracteur : l'écoulement est en équilibre géostrophique, ce qui signifie que la force de Coriolis est en équilibre avec le gradient horizontal de la pression ; un régime inertiel est constaté depuis les échelles de quelques milliers de $\mathrm{km}$ aux échelles de quelques dizaines de $\mathrm{km}$ compatible avec la turbulence bidimensionnelle quasi-géostrophique. Par ailleurs, cet attracteur est étrange, nous constatons une convergence des trajectoires vers l'attracteur, et une divergence des trajectoires sur l'attracteur. Cela implique une forte sensibilité des prévisions aux conditions initiales, paradigme de l'effet papillon introduit par Edward Lorentz en 1963. Il est aujourd'hui constaté qu'observer l'hémisphère Nord est indispensable pour la prévision à 4 jours et qu'observer le globe entier est indispensable pour la prévision à 7 jours. Des progrès substantiels sont attendus d'une compréhension géométrique de l'attracteur humide, alors que seules des descriptions de l'attracteur sec sont aujourd'hui disponibles.

\section{ASSIMILER LES DONNÉES}

La prévision du temps présuppose donc que pour prévoir l'évolution de l'écoulement atmosphérique, nous devons en déterminer les conditions initiales. Pour ce faire nous avons développé les méthodes dites d'analyse automatique mimant le travail d'interpolation fait par l'analyste, mais incapable de synthèse conceptuelle. Des progrès méthodologiques que nous devons à Lev Gandin ont permis de prendre en compte la structure statistique des erreurs des observations et de l'information a priori conduisant à la mise en œuvre opérationnelle de l'interpolation optimale par le service météorologique canadien en 1972. Le principal progrès conceptuel a alors été la prise de conscience que l'ensemble des observations passées pouvait contribuer à l'estimation de l'atmosphère à un instant donné, et que le modèle de prévision constituait le meilleur vecteur pour transporter dans le temps l'information contenue dans les observations. L'assimilation des données était née.
La méthodologie mise en œuvre pour l'assimilation des données n'est pas simple ; en effet si chacun est à même de mettre en œuvre un filtre de Kalman, la grande dimension du problème requiert des simplifications compatibles avec la physique du problème, d'où la difficulté conceptuelle.

\section{IV — QUELLES OBSERVATIONS ASSIMILER?}

La principale source d'information pour la prévision numérique est constituée par les mesures de vent, pression, température et humidité faites à partir des ballons-sondes envoyés toutes les 12 heures pour sonder l'atmosphère sur la verticale. Météo-France en exploite 7 sur la métropole et un nombre équivalent dans les départements et territoires outremer, ainsi que les TAAF. Sur les océans, les radiomètres infrarouge et hyperfréquence permettent de restituer les profils de température et d'humidité à partir des spectres observés et des radars mesurent le vent à la surface de l'océan. Les stations au sol mesurent, mais uniquement à la surface, pression, température, vent et humidité, des mesures similaires sont effectuées sur les navires volontaires de la marine marchande. L'aviation commerciale fournit des mesures au niveau de vol des avions, environ $10 \mathrm{~km}$, et lors de leur phase d'ascension et de descente au voisinage des aéroports. Des vents sont estimés à partir du déplacement des nuages observés par les satellites géostationnaires dont on voit les images à la télévision. Enfin des bouées ancrées et dérivantes permettent d'obtenir des observations à la surface des océans en dehors des routes maritimes. Cela montre le caractère composite du système d'observation en météorologie, caractère essentiel de tout réseau de mesure en géophysique.

Mais alors qu'est-ce qu'une observation ? C'est la combinaison de la mesure proprement dite, de l'opérateur permettant de relier la variable d'état du modèle au paramètre observé, et une caractérisation statistique des erreurs d'observation qui comprend les erreurs instrumentales, bien sûr, mais aussi les erreurs de représentativité liées aux échelles ou phénomènes non résolus par le modèle de prévision. La compréhension de ce qu'est ce triplet est essentielle pour une bonne utilisation des observations qui visera à extraire de façon optimale le signal géophysique présent.

\section{V — LA DYNAMIQUE DES ERREURS}

Les progrès récents en assimilation des données ont permis d'associer de façon de plus en plus étroite la dynamique de l'écoulement et l'utilisation des observations, que ce soit par l'assimilation variationnelle quadridimensionnelle ou par le développement de filtres de Kalman d'ordre réduit. L'un ou l'autre de ces algorithmes utilise une approximation de la dynamique des erreurs de prévision afin de réduire la dimensionalité formidable du problème d'estimation. Ainsi, assimilation des données et prévisibilité à courte échéance deviennent de plus en plus imbriquées, les concepts introduits par Lorenz dans ses publications de 1963 et 1965 conservant toute leur acuité. Mais des approximations pertinentes décrivant la dynamique des erreurs sur l'attracteur sont encore à explorer.

Par ailleurs, la meilleure compréhension de la propagation des erreurs permet d'envisager de concentrer les observations sur les zones instables - sources de divergence de trajectoire sur l'attracteur. Ce concept d'observation adaptative est appelé à se développer fortement dans la décennie à venir. 


\section{VI — LES DIMENSIONS D'ESPACE, DE TEMPS, ET MEME LA DIVERSITÉ DES PARAMÈTRES SE CONFONDENT DANS UNE VISION MODERNE DE LA PRÉVISION DU TEMPS}

Le paradigme fondateur de la prévision numérique du temps est issu d'une vision déterministe et par là même réductrice : les équations de la mécanique requièrent des conditions initiales pour être intégrées et ainsi conduire à une prévision. De même la prévision probabiliste qui est maintenant de plus en plus utilisée pour la prévision à 4-7 jours présume que nous devons estimer la loi de probabilité initiale de l'atmosphère avant d'intégrer l'équation déterministe de Fokker-Planck qui décrit l'évolution temporelle de cette loi de probabilité.

Bien sûr, ce paradigme est extrêmement fructueux. Ainsi nous avons développé des modèles capables de simuler l'évolution de l'écoulement atmosphérique avec une précision grandissante et reproduisant un nombre croissant de phénomènes physiques. Qui aurait pensé il y a 10 ans que les progrès de la représentation des nuages permettraient aujourd'hui de comparer quantitativement les luminances énergétiques spectrales prévues avec celles mesurées par les radiomètres embarqués sur les satellites géostationnaires et défilants ? Qui aurait pensé il y a 20 ans que les systèmes opérationnels de prévision seraient à même d'identifier que nord magnétique et nord géographique avaient été confondus sur la rose des vents d'une station météorologique d'une île isolée.

\section{VII — DE L'ESTHÉTIQUE DE NAVIER-STOKES}

Ainsi les dimensions d'espace, de temps et la dimension, discrète des paramètres sont maintenant indissociables en assimilation des observations ; le paradigme est donc d'ores et déjà remis en cause. Devons-nous aller au-delà? Je ne sais. Il est néanmoins frappant de constater que deux prévisions issues de conditions initiales différant de 6 heures sont éminemment corrélées, sans que nous en tirions parti en prévision numérique ou sans que l'on sache conceptualiser le terme d'expertise glissante. Les erreurs de prévision qui vont se développer dans les 24 heures à venir sont extrêmement sensibles à de petites modifications des conditions initiales ; les erreurs d'estimation sont concentrées sur les directions qui ont été les plus instables dans la période d'assimilation. Quel hasard fait que ces deux sous-espaces décrivant d'une part les erreurs qui ont crû et d'autre part celles qui vont croître sont suffisamment orthogonaux pour que nos prévisions aient la qualité que l'on sait ?

"Mais des figures formées au hasard ne sont que par hasard des figures harmoniques" (Valéry). 